\title{
Design and Performance Analysis of a Purely Textile Spiral Antenna for On-Body NFC Applications
}

\author{
Ruben Del-Rio-Ruiz, Juan-Manuel Lopez-Garde \\ and Jon Legarda Macon \\ DeustoTech Deusto Foundation \\ Avda Universidades, 24, Bilbao, 48007 \\ Faculty of Engineering, University of Deusto \\ Avda. Universidades, 24, Bilbao, 48007 \\ Emails: ruben.delrio@deusto.es,jmlopez@deusto.es \\ and jlegarda@deusto.es
}

\author{
Hendrik Rogier \\ Ghent University/IMEC, IDLab \\ Department of Information Technology (INTEC) \\ B-9052 Ghent, Belgium \\ Email: Hendrik.Rogier@ugent.be
}

\begin{abstract}
Novel combinations of materials and construction techniques are key for the development of new textile antenna configurations for on-body applications. Stretchable, flexible and conformable features of textile antennas are one of the hot topics in research nowadays. This work gives a step forward with new designs of purely textile spiral antennas with flexible and robust features for Near Field Communications (NFC) onbody applications. Their performance is successfully validated with a real NFC chipset, and some design and construction considerations for novel textile materials are offered.
\end{abstract}

\section{INTRODUCTION}

The design of textile antennas has gained much popularity in the last few years, specifically on new designs, textile materials and construction methodologies for new applications [1], [2]. The existing textile antenna designs are based on sewing, embroidery and gluing of conventional threads and fabrics, but new challenges arise when flexibility, stretchability, conformability and robustness characteristics become the key for on-body applications. The implementation of textile conductive fabrics, threads, paints or glues to obtain some of these properties are being used by most authors giving good results [3], [4]. Nevertheless, the combination of these textile materials with innovative designs can lead to, for example, more flexible antennas keeping their Electromagnetic (EM) characteristics.

Near field spiral antennas are highly attractive for bodycentric and wearable applications. Due to the growing of RFID technology in garments, the tag antenna design becomes one of the most interesting research topics for body centric environments. NFC and RFID tags extract their power from the field received from the reader. The efficient transfer of energy from the reader to the tag depends on the loop antenna, that must be tuned to the carrier frequency. RFID technologies are generally classified into four frequency ranges; i.e., LF (125$134.2 \mathrm{kHz}), \mathrm{HF}(13.56 \mathrm{MHz}), \mathrm{UHF}(433,860-960 \mathrm{MHz})$ and microwave frequencies $(2.45,5 \mathrm{GHz})$.
This work shows the performance and comparison of six LF square spiral antnnas (13.56 MHz) utilizing different conductive and dielectric materials. First, a reference classic spiral antenna has been designed and developed in a FR4 printed circuit board. Secondly, three designs have been developed with a conductive textile thread sewn into a flannel, a Polyester/Cottom, and a leather fabric. Finally, the performance of all antennas has been analyzed and compared, and relevant conclusions are presented.

\section{Spiral Antenna Design and Construction}

A $13.56 \mathrm{MHz}$ coil antenna has been designed. A square shape has been chosen, because of its simpler construction in textile layers. The next phase is to determine the final user application of the antenna, and its placement in the garment to choose the overall dimensions and gaps between turns. The line width of the coil antenna's will have to be as close as possible to the widths of the conductive threads, commonly between $0.1 \mathrm{~mm}$ and $0.6 \mathrm{~mm}$. The designed antenna's purpose is to be embedded in a textile bracelet by sewing a conductive thread on it.

The equivalent model of the NFC system is divided into the antenna and the chip circuits. The antenna circuit includes three components in parallel:

- $C_{a n t}$ : overall capacitance of the coil antenna.

- $R_{\text {ant }}$ : resistive loss of the coil antenna.

- $L_{a n t}$ : self inductance of the coil antenna.

The NFC chip is represented by $R_{\text {chip }}$ showing its current consumption, in parallel with $C_{t a g}$ representing its internal tuning capacitance and parasitics. The resulting antenna impedance is given by Equation 1 .

$$
Z_{\text {ant }}=C_{\text {ant }}\left|R_{\text {ant }}\right| L_{\text {ant }}
$$

The stray capacitance is generally in a range of few $\mathrm{pF}$ for typical NFC antennas and its actual value must be measured 


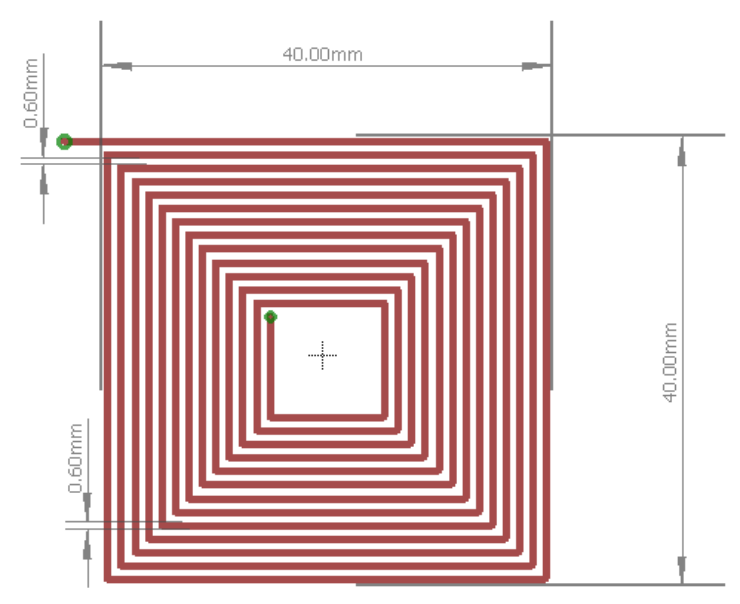

Fig. 1. Layout of the square shaped coil antenna.

once the antenna is constructed, in order to perform the fine tuning. $L_{\text {ant }}$ is calculated in two different ways. On the one hand, using Equation 2 [8].

$$
L_{\text {ant }}=K_{1} \cdot \mu_{0} \cdot N^{2} \cdot \frac{d}{1+K_{2} \cdot p}=4,63 u H
$$

On the other hand, using eDesignSuite antenna design tool, which takes into consideration more variables, such us the material's properties and thicknesses. The result obtained is $L_{\text {ant }}=5,04 u \mathrm{H}$ and it will be chosen since the difference is not too far from the one calculated in Equation 2.

In order to verify the antenna's internal capacitance and the tuning frequency, a prototype needs to be produced. A network analyzer with an ad-hoc probe and the contact-less method has been chosen to characterize the coil antennas.

Six antennas have been constructed, with 3 different types of materials. The first two antennas have been constructed on FR4 PCB boards. The other four, use purely textile layers, with the same conductive thread but different dielectric fabrics. Table II shows each antenna, named with a unique code, and their constitutive materials.

TABLE I

SPIRAL ANTENNA CONSTRUCTION MATERIALS

\begin{tabular}{|c|c|c|}
\hline Code & Conductive material & Dielectric material \\
\hline S7-1SA-B & PCB Copper & FR4 \\
\hline S7-2SA-B & 2 ply conductive thread [5] & PES/CO \\
\hline S7-3SA-B & 2 ply conductive thread [5] & Franela \\
\hline
\end{tabular}

Different conductive threads [6] [7] have been sewn in the antenna giving negative results, such us, breaking the thread while sewing, threads touching one to each other because of the thread thickness, overlapping thread on the layer and high modification of the textile's flexibility. Therefore the 2 ply conductive thread [5] has been chosen to construct the textile antennas.
In Table II and III the coil material's EM properties are shown.

TABLE II

Antennas' Conductive Materials Properties

\begin{tabular}{|c|c|c|}
\hline Material & Resistivity $\Omega /$ inch & Thickness $(\mathrm{mm})$ \\
\hline PCB Copper & 0.042 & 0.035 \\
\hline 2 ply conductive thread & 1.3 & 0.2 \\
\hline
\end{tabular}

The permittivity and loss tangent of the franela and Polyester/Cotton (PES/CO) materials shown in Table III have been obtained with the Agilent 85070E Dielectric Probe Kit.

TABLE III

Antennas' Dielectric Materials Properties

\begin{tabular}{|c|c|c|c|}
\hline Material & Permittivity & Loss Tangent & Thickness $(\mathrm{mm})$ \\
\hline FR4 & 4.7 & 0.014 & 1.2 \\
\hline PES/CO & 2 & 0.08 & 0.3 \\
\hline Franela & 1.8 & 0.06 & 0.3 \\
\hline
\end{tabular}

The next phase was to construct the PCB for the reference antenna, and the PCB textile coil holder as shown in Figure 2 and Figure 3 respectively. Both layouts include a SMA connector, and a 3 pin-out footprint (to connect the NFC chip). The layouts were performed with Autodesk Eagle PCB Design Software and the PCB were constructed with the LPKF ProtoMat S62.

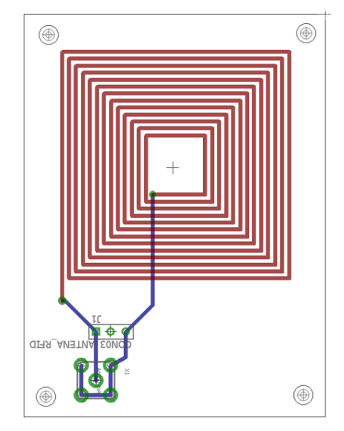

Fig. 2. PCB Layout of S7-1SA-B coil antennas.

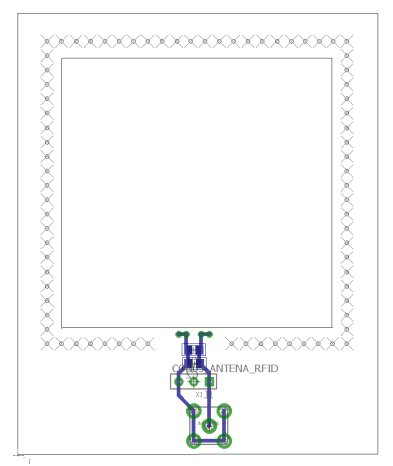

Fig. 3. PCB Layout of S7-2SA-B and S7-3SA-B coil antenna holders.
The PCB textile holder provides the textile coils with equal mechanical characteristics (flatness, and distributed tension), and easy connectivity with the SMA and 3pin-out ports, as it can be seen in Figure 3 and 4. Two PCB vias per end have been included in the textile antenna holders in order to attach the conductive threads to the PCB lines.

\section{RESULTS}

The reflection coefficient of six square spiral antennas have been characterized by using an Agilent N5242A Microwave Network Analyzer (PNA) and an ad-hoc constructed single loop antenna connected to one of the PNA ports. The coil 


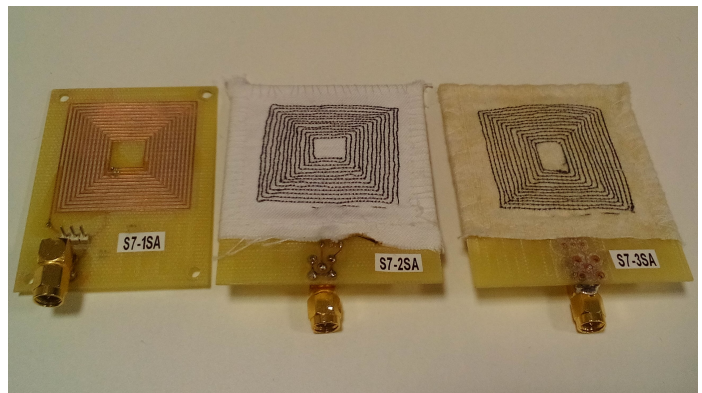

Fig. 4. Coil antennas constructed in PCB (S7-1SA), in Polyester and Cotton (PES-CO)(S7-2SA), and in franela (S7-3SA)

antennas have been tuned for their resonance frequency to be centered at $13.56 \mathrm{MHz}$. In order to do that, $C_{\text {ant }}$ has been measured, and taking into account $C_{t a g}$, an external capacitor of $6.83 \mathrm{pF}$ has been introduced.

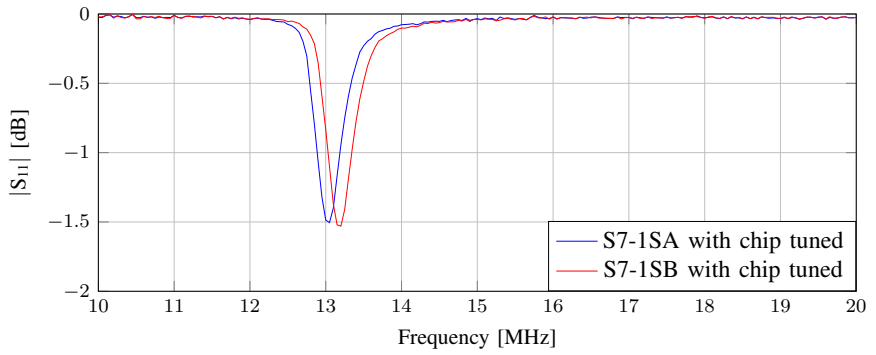

Fig. 5. Measurement results for the input reflection coefficient of the S7-S1A and S7-S1B antennas with tuning capacitor.

Antenna prototypes have been measured using $10 \mathrm{pF}$ instead of $6.83 \mathrm{pF}$, as this capacitor value was not available. Nevertheless, the actual resonant frequency of S7-1SA-B antennas, as shown in Figure 5, match if we have into account the actual $C_{\text {tun }}$ used. The resonant frequencies of S7-2SA-B and S73SA-B antennas are shifted up around 0.8 to $1 \mathrm{MHz}$ compared with the S7-1SA-B antennas, as shown in Figure 6. This means that the antenna's internal capacitance generated by the textile materials (both dielectric and conductive) is different to the S7-1SA-B loop antennas.

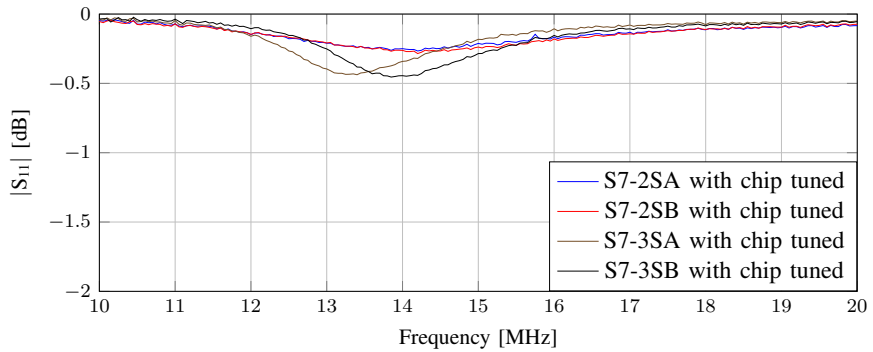

Fig. 6. Measurement results for the input reflection coefficient of the S7S2A-B and S7-S2A-B antennas with tuning capacitor.

The reflection coefficient levels of S7-3SA-B antennas have better performance $(-0.45 \mathrm{~dB})$ than the S7-2SA-B antennas ($0.25 \mathrm{~dB}$ ) due to the higher permittivity of the dielectric layers.
In order to evaluate the textile coil's performance, durability, and flexibility, it has been embedded in a leathered bracelet with a NFC chip and used in real-life operation for a year. The results obtained show that the reading time and distance is the same as commercial MIFARE contact-less (Figure 8) cards in $100 \%$ of the times even in harsh conditions (bending, crumpling, moisture environments). The prototyped bracelet is shown in Figure 7.

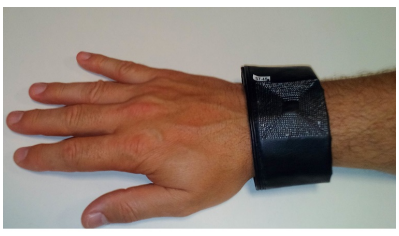

Fig. 7. Designed textile coil embedded in a leathered bracelet.

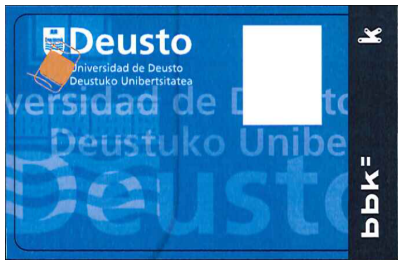

Fig. 8. MIFARE contact-less Deusto card.

\section{CONCLUSION}

The use of conductive threads sewn into franela, PES/CO and leather fabrics has been proved as a feasible construction method for NFC square spiral purely textile on-body antennas. The mechanical properties of the developed prototype, such as stress, crumpling and bending, have been successfully tested for more than one year of real life use.

Open issues for future work are related to textile connectivity procedures, stretchable designs and other novel construction methods, as ink-jet and screen printing techniques.

\section{ACKNOWLEDGMENT}

Ruben Del-Rio-Ruiz's scholarship for PhD students has been granted by the Department of Education of the Basque Government. The authors would also like to thank Textil Santanderina for their help with the manufacturing of the antennas.

\section{REFERENCES}

[1] L. Vallozzi, D. Pepe, T. Castel, H. Rogier and D. Zito, On-Body Characterization of Planar Differential Antennas for Multiple, Wide, and Narrow Bands, Volume 2016, Article ID 7439403, 9 pages. International Journal of Antennas and Propagation 2016.

[2] H. Xiaomu, S. Yan and G. A. E. Vandenbosch, Wearable Button Antenna for Dual-Band WLAN Applications With Combined on and off-Body Radiation Patterns, Volume 65, NO. 3. IEEE Transactions on Antennas and Propagation, March 2017.

[3] G. Ginestet, N. Brechet, J. Torres, E. Moradi L. Ukkonen, T. Bjrminen and J. Virkki, On-Body Characterization of Planar Differential Antennas for Multiple, Wide, and Narrow Bands, Volume 16, pag.: 1205-1208 . IEEE Antennas and Wireless Propagation Letters, 2017.

[4] T. Kaufmann, and C. Fumeaux, Wearable Textile Half-Mode SubstrateIntegrated Cavity Antenna Using Embroidered Vias, Volume 12, pag.: 805-808 . IEEE Antennas and Wireless Propagation Letters, 2013.

[5] Adafruit, Stainless Thin Conductive Thread 2 ply 23 meter/76 ft, https://www.adafruit.com/products/640 Accessed: 22/05/2017.

[6] Adafruit, Stainless Thin Conductive Yarn / Thick Conductive Thread - 35 $f t$, https://www.adafruit.com/products/603 Accessed: 22/05/2017.

[7] Adafruit, Stainless Medium Conductive Thread - 3 ply - 18 meter/60 ft, https://www.adafruit.com/products/641 Accessed: 22/05/2017.

[8] ST, How to design a $13.56 \mathrm{MHz}$ customized antenna for ST25 NFC/RFID Tags, http://bit.ly/DesigningNFCtantenna Accessed: 22/05/2017. 